International Journal of Pure and Applied Mathematics

Volume 90 No. 4 2014, 439-445

ISSN: 1311-8080 (printed version); ISSN: 1314-3395 (on-line version)

url: http://www.ijpam.eu

doi: http://dx.doi.org/10.12732/ijpam.v90i4.6

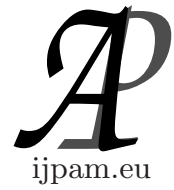

\title{
A CLASS OF SPANNED, SIMPLE AND BIUNIFORM VECTOR BUNDLES ON THE SMOOTH QUADRIC SURFACE
}

\author{
E. Ballico \\ Department of Mathematics \\ University of Trento \\ 38123 Povo (Trento) - Via Sommarive, 14, ITALY
}

\begin{abstract}
We study a class of spanned, simple and biuniform vector bundles on a smooth quadric surface $Q$ (the duals of general surjections $\mathcal{O}_{Q}^{(r+1)} \rightarrow$ $\left.\mathcal{O}_{Q}(a, b)\right)$.
\end{abstract}

AMS Subject Classification: $14 J 60$

Key Words: spanned vector bundle, quadric surface, biuniform vector bundle

\section{Introduction}

Let $Q:=\mathbb{P}^{1} \times \mathbb{P}^{1} \subset \mathbb{P}^{3}$ be a smooth quadric surface. In this note we study the following family of spanned vector bundles on $Q$. Fix positive integers $a, b, r$ such that $2 \leq r \leq a b+a+b$. Let $S(a, b, r)$ be the set of all surjective $\operatorname{maps} \mathcal{O}_{Q}^{\oplus(r+1)} \rightarrow \mathcal{O}_{Q}(a, b)$. Since $r+1 \geq 3>\operatorname{dim}(Q)$, a dimensional count gives that the set $S(a, b, r)$ is a non-empty open subset of the vector space $H^{0}\left(\mathcal{O}_{Q}(a, b)^{\oplus(r+1)}\right)$ and hence it is an integral variety of dimension $(a+1)(b+$ $1)(r+1)$. For each $\phi \in S(a, b, r)$ the sheaf $\operatorname{ker}(\phi)$ is a vector bundle on $Q$ with determinant isomorphic to $\mathcal{O}_{Q}(-a,-b)$. The sheaf $\operatorname{ker}(\phi)$ is a subsheaf

Received: September 5, 2013

(c) 2014 Academic Publications, Ltd. url: www.acadpubl.eu 
of $\mathcal{O}_{Q}^{\oplus(r+1)}$ and $\mathcal{O}_{Q}^{\oplus(r+1)} / \operatorname{ker}(\phi) \cong \mathcal{O}_{Q}(a, b)$. Since the dual of a short exact sequence of vector bundles is exact, the vector bundle $\operatorname{ker}(\phi)^{\vee}$ is spanned. Let $G(a, b, r)$ be the set of all vector bundles $\left\{\operatorname{ker}(\phi)^{\vee}\right\}_{\phi \in S(a, b, r)}$. Any $E \in G(a, b, r)$ has rank $r, c_{1}(E)=(a, b)$, and it is spanned by its global sections. Since $E$ is spanned, then $h^{2}(E)=h^{0}\left(E^{\vee}(-2,-2)\right)=0$. It fits in an exact sequence

$$
0 \rightarrow \mathcal{O}_{Q}(-a,-b) \rightarrow \mathcal{O}_{Q}^{\oplus(r+1)} \rightarrow E \rightarrow 0 .
$$

Since $a>0$ and $b>0$, we have $h^{i}\left(\mathcal{O}_{Q}(-a,-b)\right)=0, i=0,1$. Hence $h^{0}(E)=$ $r+1$ and $h^{1}(E)=h^{2}\left(\mathcal{O}_{Q}(-a,-b)\right)=(a-1)(b-1)$. We may do the same definition even if $r \geq(a+1)(b+1)$, but if $r \geq(a+1)(b+1)$, then $E \in G(a, b, r)$ $\Leftrightarrow E \cong F \oplus \mathcal{O}_{Q}^{\oplus(r-a b-a-b)}$ for some $F \in G(a, b, a b+a+b)$. Assume again $2 \leq r \leq a b+a+b$. Let $S^{\prime}(a, b, r)$ be the set of all $f \in S(a, b, r)$ associated to $r+1$ linearly independent sections of $\mathcal{O}_{Q}(a, b)$. Let $G^{\prime}(a, b, r)$ the the set of all vector bundles $\left\{\operatorname{ker}(\phi)^{\vee}\right\}_{\phi \in S^{\prime}(a, b, r)}$. It is easy to check that $E \in G^{\prime}(a, b, r)$ if and only if $E \in G(a, b, r)$ and $\mathcal{O}_{Q}$ is not a factor of $E$.

Let $E$ be a rank $r$ vector bundle on $Q$. We say that $E$ is biuniform if there are integers $a_{1} \geq \cdots \geq a_{r}$ and $b_{1} \geq \cdots \geq b_{r}$ such that $E \mid D$ has splitting type $\left(a_{1}, \cdots, a_{r}\right)$ for all $D \in\left|\mathcal{O}_{Q}(0,1)\right|$ and splitting type $\left(b_{1}, \ldots, b_{r}\right)$ for all $T \in\left|\mathcal{O}_{Q}(1,0)\right|([1])$. We say that a splitting type $c_{1} \geq \cdots \geq c_{r}$ is balanced if $c_{1} \leq c_{r}+1$. In this note we prove the following result.

Theorem 1. Assume $2 \leq r \leq a b+a+b$.

1. Every $E \in G^{\prime}(a, b, r)$ is simple.

2. Assume that neither a nor $b$ is divisible by $r$. Then a general $E \in G(a, b, r)$ is biuniform with balanced splitting types.

Part 1 easily follows from the fact that the spanned bundle $E$ has no trivial factor and that $\operatorname{rank}(E)=h^{0}(E)-1$ (see step (a) of the proof of Theorem 1). Part 2 is related to the following result concerning simple bundles. For all $c_{1} \in \operatorname{Pic}(Q), c_{2} \in \mathbb{Z}$ and $r \geq 2$ let $\mathcal{S}\left(c_{1}, c_{2} ; r\right)$ denote the moduli space of all rank $r$ simple vector bundles $F$ on $Q$ with $c_{1}(F)=c_{1}$ and $c_{2}(F)=c_{2}$. It exists ([3], [4]), although it may be empty for some $c_{1}, c_{2}, r$. It is everywhere smooth (Remark 2). We need to adapt to the bundles in $G^{\prime}(a, b, r)$ a proof of the following well-known result (true because the anticanonical line bundle $\omega_{Q}^{\vee} \cong \mathcal{O}_{Q}(2,2)$ is ample and spanned).

Proposition 1. Fix $c_{1}, c_{2}, r$ such that $r \geq 2$ and $\mathcal{S}\left(c_{1}, c_{2}, r\right) \neq \emptyset$. Take $c_{1}=(a, b)$ and assume that neither $a$ nor $b$ is divisible by $r$. Let $\mathcal{S}$ be any connected component of $\mathcal{S}\left(c_{1}, c_{2}, r\right)$. Then a general $E \in \mathcal{S}$ is biuniform. 
When $b$ is large we also show how to construct many non-biuniform vector bundles $E \in G^{\prime}(a, b, r)$ with prescribed splitting type at several elements of $\left|\mathcal{O}_{Q}(0,1)\right|$ (Proposition 2).

Remark 1. There is a unique bundle $E \in G^{\prime}(a, b, a b+a+b)$ (up to isomorphisms), because any $E \in G^{\prime}(a, b, a b+a+b)$ is induced by the complete linear system $\left|\mathcal{O}_{Q}(a, b)\right|$. Hence $f^{*}(E) \cong E$ for all $f \in \operatorname{Aut}\left(\mathbb{P}^{1}\right) \times \operatorname{Aut}\left(\mathbb{P}^{1}\right)$ (the connected component of the identity of the algebraic group $\operatorname{Aut}(Q))$. If $a=b$, then $\sigma^{*}(E) \cong E$ for the order two automorphism of $Q$ which exchanges the two rulings of $Q$. Take $r \geq(a+1)(b+1)$ and a general $A \in G(a, b, r)$. Since $A \cong E \oplus \mathcal{O}_{Q}^{\oplus(r-a b-a-b)}$ for some $E \in G^{\prime}(a, b, a b+a+b)$, we get that $A$ is biuniform (but not simple).

\section{The Proof}

We fix integers $a, b, r$ such that $a>0, b>0$ and $2 \leq r \leq a b+a+b$. Let $A$ be a rank $r$ vector bundle on $\mathbb{P}^{1}$. The splitting type of $A$ is balanced if and only if $h^{1}\left(A \otimes A^{\vee}\right)=0$.

Lemma 1. Let $E$ be a simple vector bundle on $Q$. Then $h^{2}(\operatorname{End}(E)(c, d))=$ 0 for all integers $c \geq-1$ and $d \geq-1$.

Proof. Since $E$ is simple, every element of $H^{0}(\operatorname{End}(E))$ is induced by the multiplication by a scalar. Hence $h^{0}(\operatorname{End}(E)(-2-c,-2-d))=0$ for all $c \geq-1$ and $d \geq-1$.

Remark 2. Let $E$ be a simple vector bundle. Set $c_{i}:=c_{i}(E)$ and $r:=$ $\operatorname{rank}(E)$. Since $h^{2}(E n d(E))=0$ (Lemma 1), $\mathcal{S}\left(c_{1}, c_{2}, r\right)$ is smooth at $E$ and of dimension $h^{1}(\operatorname{End}(E))$.

Proof of Proposition 1. Since $\mathcal{S}$ is smooth (Remark 2) and connected, it is irreducible. Hence it is sufficient to prove the existence of non-empty open subsets $U_{1}$ and $U_{2}$ of $\mathcal{S}$ such that each $E_{i} \in U_{i}$ is uniform with respect to the ruling of $Q=\mathbb{P}^{1} \times \mathbb{P}^{1}$ corresponding to the $i$-th projection $Q \rightarrow \mathbb{P}^{1}$. Just to fix the notation we prove the existence of this open subset of $\mathcal{S}$ with respect to the projection $\pi: Q \rightarrow \mathbb{P}^{1}$ such that $\mathcal{O}_{Q}(0,1) \cong \pi^{*}\left(\mathcal{O}_{\mathbb{P}^{1}}(1)\right)$. Write $c_{1}=$ $\mathcal{O}_{Q}(u, v)$. By assumption $u / r \notin \mathbb{Z}$. For all $u_{1} \geq \cdots \geq u_{r}$ such that $u_{1}+\cdots+u_{r}$ and each $D \in\left|\mathcal{O}_{Q}(0,1)\right|$ let $\mathcal{S}\left(D ; u_{1}, \ldots, u_{r}\right)$ be the set of all $E \in \mathcal{S}$ such that $E \mid D$ has splitting type $u_{1}, \ldots, u_{r}$. Since $\mathcal{S}$ is an algebraic variety, the semicontinuity theorem for cohomology gives that $\mathcal{S}\left(D ; u_{1}, \ldots, u_{r}\right) \neq \emptyset$ only for 
finitely many splitting types. Since $\operatorname{dim}\left(\left|\mathcal{O}_{Q}(0,1)\right|\right)=1$, to conclude the proof it is sufficient to prove that for each unbalanced splitting type $u_{1}, \ldots, u_{r}$ and for each $D \in\left|\mathcal{O}_{Q}(0,1)\right|$ the set $\mathcal{S}\left(D ; u_{1}, \ldots, u_{r}\right)$ has codimension at least two in $\mathcal{S}$. Set $F:=\oplus_{i=1}^{r} \mathcal{O}_{D}\left(u_{i}\right)$ and call $\mathcal{U}$ a versal deformation space of $F$ (smooth of dimension $h^{1}(D, E n d(F))$ with a prescribed point $o \in \mathcal{U}$ corresponding to $F$. Since $u / r \notin \mathbb{Z}$ and $u_{1}, \ldots, u_{r}$ is unbalanced, we have $h^{1}(D, \operatorname{End}(F)) \geq 2$. Fix any $E \in \mathcal{S}\left(D ; u_{1}, \ldots, u_{r}\right)$. Since $h^{2}(E(0,-1))=0$ (Lemma 1$)$, the restriction $\operatorname{map} H^{1}(\operatorname{End}(E)) \rightarrow H^{1}(D, \operatorname{End}(F))$ is surjective. Hence $\mathcal{S}\left(D ; u_{1}, \ldots, u_{r}\right)$ has codimension $h^{1}(D, \operatorname{End}(F))$ in $\mathcal{S}$. Since $h^{1}(D, \operatorname{End}(F)) \geq 2$, we are done.

Lemma 2. Fix $D \in\left|\mathcal{O}_{Q}(0,1)\right|$ and integers $a_{1} \geq \cdots \geq a_{r} \geq 0$ such that $a_{1}+\cdots+a_{r}=a$. Set $F:=\oplus_{i=1}^{r} \mathcal{O}_{\mathbb{P}^{1}}\left(a_{i}\right)$. Let $A$ be the set of all surjections $\psi: \mathcal{O}_{\mathbb{P} 1}^{\oplus(r+1)} \rightarrow \mathcal{O}_{\mathbb{P}^{1}}(a)$ such that $\operatorname{ker}(\psi) \cong F^{\vee}$. Then $A \neq \emptyset$. Fix $\psi \in A$. Let $B$ (resp. $\left.B^{\prime}\right)$ be the set of all $E \in G(a, b, r)$ (resp. $E \in G^{\prime}(a, b, r)$ ) of the form $\operatorname{ker}(u)^{\vee}$ with $u: \mathcal{O}_{Q}^{\oplus(r+1)} \rightarrow \mathcal{O}_{Q}(a, b)$ a surjection and $u \mid D=\psi$. Then $B$ is an irreducible variety of dimension $(r+1)(a+1) b$ and $B^{\prime}$ is a non-empty Zariski open subset of $B$.

Proof. Every spanned vector bundle $G$ on an integral variety $T$ is spanned by at most $\operatorname{dim}(T)+\operatorname{rank}(G)$ sections (wait a few lines for the proof of this claim). Hence $A \neq \emptyset$. Fix $\psi \in A$. Let $B^{\prime \prime}$ be the set of all maps $f: \mathcal{O}_{Q}^{r+1} \rightarrow$ $\mathcal{O}_{Q}(a, b)$ such that $a \mid D=\psi$. Since $b>0, B^{\prime \prime} \neq \emptyset$ and $B^{\prime \prime}$ is an irreducible variety of dimension $(r+1) \cdot h^{0}\left(\mathcal{O}_{Q}(a, b-1)\right)=(r+1)(a+1) b$. Notice that $B$ is the set of all surjective elements of $B^{\prime \prime}$. Every $f \in B^{\prime \prime}$ is surjective at the points of $P$. Fix $P \in Q \backslash D$. Set $B_{P}:=\left\{f \in B^{\prime \prime}: f(P) \equiv 0\right\}$. Since $\mathcal{O}_{Q}(a, b-1)$ is spanned, $B_{P}$ has codimension $r+1$ in $B^{\prime \prime}$. Since $\operatorname{dim}(Q)=2<r+1$, we get $B \neq \emptyset$. If $a_{r}>0$, then $B^{\prime}=B$. Now assume $a_{r}=0$ and that $B^{\prime}=\emptyset$. We get that all vector bundles associated to some element of $B$ have $\mathcal{O}_{Q}$ as a factor. Taking out the factors $\mathcal{O}_{D}\left(a_{r}\right)=\mathcal{O}_{D}$ from $F$, we win counting the dimension for the data $a, b, r^{\prime}:=r-1$ and $F^{\prime}:=\oplus_{i=1}^{r-1} \mathcal{O}_{\mathbb{P}^{1}}\left(a_{i}\right)$, if we can do the case $r=2$. Assume the existence of $E \in\left(G(a, b, 2) \backslash G^{\prime}(a, b, 2)\right)$ and write $E \cong G \oplus \mathcal{O}_{Q}$. We get $G \cong \mathcal{O}_{Q}(a, b)$ and hence $c_{2}(E)=0$, a contradiction.

Let $S(a ; r)$ be the set of all surjective maps $\mathcal{O}_{\mathbb{P}^{1}}^{\oplus(r+1)} \rightarrow \mathcal{O}_{\mathbb{P}^{1}}(a)$. Since $r+1 \geq 3>\operatorname{dim}\left(\mathbb{P}^{1}\right)$, the set $S(a ; r)$ is a non-empty open subset of the vector space $H^{0}\left(\mathcal{O}_{\mathbb{P}^{1}}(a)^{\oplus(r+1)}\right)$ and hence it is an integral variety of dimension $(a+$ $1)(r+1)$. For each $\phi \in S(a ; r)$ the sheaf $\operatorname{ker}(\phi)$ is a vector bundle on $\mathbb{P}^{1}$ with determinant isomorphic to $\mathcal{O}_{\mathbb{P}^{1}}(-a)$. The sheaf $\operatorname{ker}(\phi)$ is a subsheaf of $\mathcal{O}_{\mathbb{P}^{1}}^{\oplus(r+1)}$ and $\mathcal{O}_{\mathbb{P}^{1}}^{\oplus(r+1)} / \operatorname{ker}(\phi) \cong \mathcal{O}_{\mathbb{P}^{1}}(a)$. Since the dual of a short exact sequence of 
vector bundles is exact, the vector bundle $\operatorname{ker}(\phi)^{\vee}$ is spanned. Let $G(a ; r)$ be the flat family $\left\{\operatorname{ker}(\phi)^{\vee}\right\}_{\phi \in S(a ; r)}$ of vector bundles on $\mathbb{P}^{1}$. Any $E \in G(a ; r)$ has rank $r$ and it is spanned. Hence the splitting type $a_{1} \geq \cdots \geq a_{r}$ of any $E \in G(a ; r)$ satisfies the conditions $a_{r} \geq 0$ and $a_{1}+\cdots+a_{r}=a$.

Lemma 3. Fix integers $a_{1} \geq \cdots \geq a_{r}$ such that $a_{1}+\cdots+a_{r}=a$ and set $F:=\oplus_{i=1}^{r} \mathcal{O}_{\mathbb{P}^{1}}\left(a_{i}\right)$. The set of all $E \in G(a ; r)$ isomorphic to $F$ is non-empty and it has dimension $(r+1)(a+1)-h^{1}(\operatorname{End}(F))$.

Proof. We have $h^{0}(F)=a+r$. Hence the Grassmannian of all $(r+1)$ dimensional linear subspaces of $H^{0}(F)$ has dimension $(r+1)(a-1)$. For any rank $r$ vector bundle $A$ on $\mathbb{P}^{1}$ we have $\chi(\operatorname{End}(A))=r^{2}$, because $\operatorname{End}(A)$ has rank $r^{2}$ and degree 0 . Hence $h^{0}(\operatorname{End}(F))=h^{1}(\operatorname{End}(F))+r^{2}$. We have $\operatorname{dim}(\operatorname{Aut}(F))=h^{0}(\operatorname{End}(F))$. Two injective maps with locally free cokernels $u_{i}: F \rightarrow \mathcal{O}_{\mathbb{P} 1}^{\oplus(r+1)}, i=1,2$, have the same image if and only if $u_{2}=u_{1} \circ w$ for some automorphism $w$ of $F$.

We leave to the reader the proof of the following elementary lemma.

Lemma 4. Let $T$ be an integral projective variety. Let $E$ be a rank $r$ spanned torsion free sheaf on $T$. $E$ is a trivial vector bundle if and only if it is spanned by an $r$-dimensional linear subspace of $H^{0}(E)$.

Proof of Theorem 1. Fix $E \in G^{\prime}(a, b, r)$ (it exists, because $\left.r \leq a b+a+b\right)$. Since $E$ is spanned and without trivial factors, we have $h^{0}\left(E^{\vee}\right)=0$, i.e. there is no non-zero map $E \rightarrow \mathcal{O}_{Q}$.

(a) Assume that $E$ is not simple. Hence there is a non-zero map $f: E \rightarrow$ $E$ such that $G:=\operatorname{Im}(f)$ has rank $m<r$. Since $G \subset E, G$ is torsion free. Let $V \subseteq H^{0}(G)$ be the image of the map $u: H^{0}(E) \rightarrow H^{0}(G)$ induced by the surjection $E \rightarrow G$. Since $G=f(E)$ is a quotient of the spanned sheaf $E$, the sheaf $G$ is spanned by $V$. Set $W:=\operatorname{ker}(u) \subseteq H^{0}(E)$. Since every map $E \rightarrow \mathcal{O}_{Q}$ is trivial, $G$ is not a trivial vector vector bundle. Hence $\operatorname{dim}(V) \geq m+1$ (Lemma 4). Hence $\operatorname{dim}(W) \leq r-m$. Let $A$ be the saturation of $G$ in $E$, i.e. the only rank $m$ subsheaf of $E$ containing $G$ and such that $E / A$ has no torsion. The sheaf $E / A$ has rank $r-m$. Since $E / A$ is a quotient of $E / G$, it is spanned by a quotient of $W$. Since $\operatorname{dim}(W) \leq r-m$, the torsion free sheaf $E / A$ is a trivial vector bundle (Lemma 4). Hence $E$ has $\mathcal{O}_{Q}^{r-m}$ as a factor, a contradiction.

(b) In this step we assume that neither $a$ nor $b$ is divisible by $r$ and prove that a general $E \in G^{\prime}(a, b, r)$ is biuniform. We adapt the proofs in [2]. We fix one of the two rulings of $Q$, say $\left|\mathcal{O}_{Q}(0,1)\right|$, since the proof for the other ruling is similar. Fix $D \in\left|\mathcal{O}_{Q}(0,1)\right|$. Fix integers $a_{1} \geq \cdots \geq a_{r} \geq 0$ such 
that $a_{1}+\cdots+a_{r}=a$ and $a_{r} \leq a_{1}-2$. Call $u: G^{\prime}(a, b, r) \times\left|\mathcal{O}_{Q}(0,1)\right| \rightarrow$ $\left|\mathcal{O}_{Q}(0,1)\right|$ the projection onto the second factor. Let $\Gamma\left(a_{1}, \ldots, a_{r}\right)$ be the set of all $(E, D) \in G^{\prime}(a, b, r) \times\left|\mathcal{O}_{Q}(0,1)\right|$ such that $E \mid D$ has splitting type $\left(a_{1}, \ldots, a_{r}\right)$. We have $\Gamma\left(a_{1}, \ldots, a_{r}\right)=\emptyset$ if $a_{r}<0$. Hence we only have finitely may nonempty sets $\Gamma\left(a_{1}, \ldots, a_{r}\right)$. Since $\operatorname{dim}\left(\left|\mathcal{O}_{Q}(0,1)\right|\right)=1$, to prove that a general $E \in G^{\prime}(a, b, r)$ is uniform with respect to the ruling associated to $\left|\mathcal{O}_{Q}(0,1)\right|$ it is sufficient to prove that each non-empty $\Gamma\left(a_{1}, \ldots, a_{r}\right)$ has codimension $\geq 2$ in $G^{\prime}(a, b, r) \times\left|\mathcal{O}_{Q}(0,1)\right|$. Fix $D \in\left|\mathcal{O}_{Q}(0,1)\right|$. It is sufficient to prove that $\operatorname{dim}\left(u^{-1}(D)\right) \leq \operatorname{dim}\left(G^{\prime}(a, b, r)\right)-2$. Set $F:=\oplus_{i=1}^{r} \mathcal{O}_{D}\left(a_{i}\right)$. Lemmas 2 and 3 give that $u^{-1}(D)$ has dimension $\operatorname{dim}\left(G^{\prime}(a, b, r)\right)-h^{1}(\operatorname{End}(F))$. Since $F$ is unbalanced and $a / r \notin \mathbb{Z}$, we have $h^{1}(\operatorname{End}(F)) \geq 2$.

Lemma 5. Let $E$ be a rank $r$ vector bundle on $Q$. Fix $T \in\left|\mathcal{O}_{Q}(1,0)\right|$ and let $b_{1} \geq \cdots \geq b_{r}$ be the splitting type of $E \mid T$. Assume the existence of $c \in \mathbb{Z}$ such that $E \mid D$ has splitting type $(c, \ldots, c)$ for all $D \in\left|\mathcal{O}_{Q}(0,1)\right|$. Then $E \cong \oplus_{i=1}^{r} \mathcal{O}_{Q}\left(c, b_{i}\right)$.

Proof. Taking $E(-c, 0)$ instead of $E$ we reduce to the case $c=0$. Let $\pi: Q \rightarrow \mathbb{P}^{1}$ denote the projection such that $\mathcal{O}_{Q}(0,1) \cong \pi^{*}\left(\mathcal{O}_{\mathbb{P}^{1}}(1)\right)$. Since $h^{0}(E \mid D)=r$ and $h^{1}(E \mid D)=0$ for every fiber $D$ of $\pi$, a theorem of base change gives that $\pi_{*}(E)$ is a rank $r$ vector bundle ([5], page 11). We have $\pi_{*}(E) \cong \oplus_{i=1}^{r} \mathcal{O}_{\mathbb{P}^{1}}\left(d_{i}\right)$ for some integers $d_{1} \geq \cdots \geq d_{r}$. For each fiber $D$ of $\pi$ the natural map $H^{0}\left(D, \pi^{*}\left(\pi_{*}(E)\right) \mid D\right) \rightarrow H^{0}(D, E \mid D)$ is an isomorphism ([5], page 11). Since $E \mid D$ is trivial, we get that the natural map $\pi^{*}\left(\pi_{*}(E)\right) \rightarrow E$ is an isomorphism (see [5], page 53, for a similar proof). Since $E \mid T$ has splitting type $b_{1} \geq \cdots \geq b_{r}$, we get $d_{i}=b_{i}$ for all $i$.

Remark 3. Lemma 5 shows that if either $a / r \in \mathbb{Z}$ or $b / r \in \mathbb{Z}$, then every biuniform vector bundle with balanced splitting type is isomorphic to a direct sum of $r$ line bundles.

Proposition 2. Fix positive integers $a, b, r, c$, such that $r \geq 2, b>c$, $a_{1}(i) \geq \cdots \geq a_{r}(i) \geq 0,1 \leq i \leq c$, and $a_{1}(i)+\cdots+a_{r}(i)=a$ for all $i \in\{1, \ldots, c\}$. Fix $D_{i} \in\left|\mathcal{O}_{Q}(0,1)\right|, 1 \leq i \leq c$, with $D_{i} \neq D_{h}$ for all $i \neq h$,. Then there is $E \in G^{\prime}(a, b, r)$ such that $E \mid D_{i}$ has splitting type $a_{1}(i) \geq \cdots \geq a_{r}(i)$ for all $i$.

Proof. Set $T:=D_{1} \cup \cdots \cup D_{c}$. Let $G$ be the vector on $D$ such that $G \mid D_{i}=\mathcal{O}_{D_{i}}\left(a_{h}(i)\right)$ for all $i$. Fix a surjection $\psi: \mathcal{O}_{T}^{\oplus(r+1)} \rightarrow G$ (it exists, because $G$ is spanned and $\operatorname{dim}(T)+\operatorname{rank}(G)=r+1)$. Since $b-c>0$, the proof of Lemma 2 works with $T$ instead of $D$. 
Assume $a \geq 2$ and $b \geq 2$ and fix integers $a_{1} \geq \cdots \geq a_{r} \geq 0$ such that $a_{1}+\cdots+a_{r}=a+b$. Fix a smooth $T \in\left|\mathcal{O}_{Q}(1,1)\right|$. The proof of Proposition 2 shows the existence of $E \in G^{\prime}(a, b, r)$ such that $E \mid T$ has splitting type $a_{1}, \ldots, a_{r}$.

\section{Acknowledgements}

The author was partially supported by MIUR and GNSAGA of INdAM (Italy).

\section{References}

[1] E. Ballico and P. E. Newstead, Uniform bundles on quadric surfaces and some related varieties, J. London Math. Soc. (2) 31 (1985), no. 2, 211-223.

[2] J. Brun and A. Hirschowitz, Droites de saut des fibrés stables de rang élevé sur $\mathbb{P}^{2}$, Math. Z. 181 (1982), no. 2, 171-178.

[3] S. Kosarew and C. Okonek, Christian Global moduli spaces and simple holomorphic bundles, Publ. Res. Inst. Math. Sci. 25 (1989), no. 1, 1-19.

[4] C. Lübke and C. Okonek, Moduli spaces of simple bundles and HermitianEinstein connections, Math. Ann. 276 (1987), no. 4, 663-674.

[5] C. Okonek, M. Schneider and H. Spindler, Vector Bundles on Complex Projective Spaces, Birkhäuser, Boston, Basel, Stuttgart, 1980. 
